\title{
Effects of good form and spatial frequency on global precedence
}

\author{
LINDA L. LAGASSE \\ University of Massachusetts, Amherst, Massachusetts
}

\begin{abstract}
Does the global precedence effect depend on the goodness of the global form and low spatial frequencies? In Experiments 1 and 2, under a variety of attentional and task conditions, a global advantage in response time (RT) occurred in "good," many-element compound patterns but not in "poor," few-element patterns (unless the local elements were too small to be easily recognized). Symmetric interference effects were found in all patterns, however, suggesting that global and local information were encoded in parallel and that the global advantage in RT involved some postperceptual processes. Experiments $3 \mathrm{~A}$ and $3 \mathrm{~B}$ showed that the global advantage in RT and perceived pattern goodness depend on low spatial frequencies: Lowpass-filtered patterns rated as "good" showed the usual global advantage in RT, but highpass-filtered, many-element forms rated as "poor" did not. These findings suggest that a global advantage in RT requires an unambiguous global form conveyed by low spatial frequencies.
\end{abstract}

An important issue in visual perception is how we come to recognize objects and patterns derived from particular arrangements of their parts. Gestalt psychologists have long suggested that the perceptual coherence and dominance of wholes results from basic organizing principles such as proximity, similarity, good continuation, and closure (Koehler, 1947). From this view, the goodness of the global form determines whether a pattern or its parts would be recognized first. More current work has shown that good form benefits all stages of information processing. For example, good form facilitates encoding (Bell \& Handel, 1976; Garner \& Sutliff, 1974) as well as memory and comparison processes (Checkosky \& Whitlock, 1973).

Unlike the Gestalt view, recent work by Navon (1977, $1981,1991)$ has suggested a different explanation for the priority of wholes over parts. Navon's primary hypothesis is that the pattern or global level of a form is always encoded faster than is more local information and hence is available sooner to recognition and response processes. The probable source for this temporal advantage is the operation of fast-conducting, visual mechanisms tuned to the low spatial frequencies of the global form (Navon, 1981; Petersik, 1978). Thus, according to this hypothe-

The research and preparation of the manuscript were supported by NICHD Grant HD05331 to Peter D. Eimas and by NIMH Post-Doctoral Training Grant MH16745 to Linda L. LaGasse. This research was based on a dissertation submitted in partial fulfillment of a doctoral degree at Brown University. I want to thank my advisor, Peter Eimas, for his many contributions to this study. Practical assistance was also provided by Terry Au, Einar Siqueland, David Knill, Philippe Schyns, and Kathy Spoehr. I am further indebted to Sandy Pollatsek for his ideas during the preparation of this manuscript, and to Lester Krueger, Michael Venturino, and an anonymous reviewer for their helpful comments. Correspondence should be addressed to Linda L. LaGasse, Infant Development Unit, Women and Infants' Hospital, 101 Dudley St., Providence, RI 02905 . sis, the low spatial frequencies of global patterns-not their good form-mediate the advantage of wholes over parts.

The present study examines whether either or both of these explanations can account for the priority of wholes. The good-form hypothesis is tested alone in Experiments $1 \mathrm{~A}, 1 \mathrm{~B}$, and 2 , and with the spatial frequency hypothesis in Experiments $3 \mathrm{~A}$ and 3B. Two additional issues are explored in Experiments 1A and 2: (1) the effect of task demands and attentional constraints on global precedence and (2) the encoding hypothesis put forth by Navon (1977). These issues are included to address some of the controversies concerning the methodology and interpretation of Navon's results. Before reviewing relevant studies on the effects of good form, an overview of the methodological and theoretical basis of the global precedence effect is presented.

Background Studies of the Global Precedence Effect

The global precedence hypothesis has mainly been explored with compound, hierarchical patterns (see Figure 1 for examples). A compound pattern consists of a large (global) form made of small (local) forms. Typically, all possible combinations of global and local shapes are used.

Navon's early work $(1977,1981)$ utilized a selectiveattention task in which subjects had to identify the form at either the global or local level. In addition, the stimulus was presented for a brief time in any of four locations in the parafovea and was immediately followed by a mask. The restrictive task conditions used by Navon elicited concern that his results did not represent a general processing advantage for the global pattern. Some subsequent studies have reported that global precedence is dependent on the method of stimulus presentation, such as brief presentation time (Paquet \& Merikle, 1984) in the parafovea (Grice, Canham, \& Boroughs, 1983; Pomerantz, 1983). 
But other studies have indicated that the global precedence effect is not dependent on Navon's initial conditions. For example, global precedence has been found with unlimited exposure time (Miller, 1981; Pomerantz, 1983), without a mask (Hoffman, 1980; Kinchla \& Wolfe, 1979; Pomerantz, 1983), in a divided-attention task (Miller, 1981; Navon, 1983; Navon \& Norman, 1983), and with foveal presentation (Navon \& Norman, 1983).

Although global precedence appears to be robust across many tasks and attentional conditions, this is not a foregone conclusion. Thus, Experiments 1A, 1B, and 2 will examine whether the association between good form and global precedence is subject to task and attentional conditions.

\section{The Encoding Hypothesis}

In early studies, a global precedence effect was defined by two phenomena: (1) global information was processed on average faster than was the local information, and (2) conflicting information on the global level interfered with discrimination on the local level, but not vice versa (Martin, 1979; Navon, 1977, 1981; Pomerantz, 1983). That is, a square on the global level interfered more with processing a diamond on the local level than vice versa. The overall response time (RT) advantage is consistent with the perceptual dominance of the pattern as opposed to the parts. However, the co-occurrence of asymmetric interference favoring the global level best supports Navon's claim for a fixed global-to-local order of processing, since it appears to indicate that global processing is not only faster on average than is local processing but occurs prior to it.

An alternative explanation for Navon's (1977) faster global processing is that, all other factors being equal, the global form is more salient (i.e., more readily attended to) hence more likely to be used first (Pomerantz, 1983; Ward, 1982). This view is mainly supported by evidence that global and local information are encoded in parallel (Boer \& Keuss, 1982; Miller, 1981). The purported postperceptual locus for global precedence is not wellspecified but usually involves attention or decision/response processes.

The encoding hypothesis is further challenged if the RT advantage for global information does not co-occur with asymmetric interference favoring the global. Navon and Norman (1983) showed an overall global advantage in RT, but there were equivalent amounts of interference from conflicting information on either level. Although these findings suggest that global and local information were encoded in parallel, Navon and Norman argued that fast, low-frequency input favored early confirmation of the global shape (global advantage in RT) while partially encoding the local shape as well (symmetric interference). According to this view, the global advantage arises from faster global encoding and faster global decisions. Thus, global processing benefits from both encoding and postperceptual processes.
Efforts to understand global precedence by interpreting the interference effects may be wholly misdirected. Lamb and Robertson (1989) showed that a global advantage in RT and observed interference effects are independent effects. Furthermore, they arise from different brain areas (Robertson, Lamb, $\&$ Knight, 1988). Patients with lesions located in the superior temporal gyrus showed difficulty with discrimination processes associated with interlevel interference, whereas patients with lesions in the inferior parietal lobe showed deficits in spatial attention linked to global processing time (Robertson et al., 1988).

In sum, the encoding explanation for global precedence is disputed by evidence for parallel encoding of global and local information and by divergence between a global advantage in RT and observed interference effects. To avoid confusion, the term "global precedence effect" will henceforth be reserved for the theoretical processing priority for global information; the term "global advantage" will refer to the observed RT advantage for global decision and will serve as the primary dependent measure. Measures of interference between levels are evaluated separately with respect to their concordance with various conceptualizations of their association to the observed global advantage.

\section{Good Form and Global Precedence}

One stimulus factor that could either facilitate encoding or attract attention to the global form is an emergent Gestalt property (Hoffman, 1980; Ward, 1983). In this case, any manipulation that reduced the goodness of form in the Gestalt sense may also eliminate a global advantage. Some studies have demonstrated that a global advantage is eliminated if the local elements were sparse (Martin, 1979; but see Navon, 1983) or if the arrangement of local elements was distorted (Hoffman, 1980). Note that such changes typically undermine the good form of the configuration (Koehler, 1947). However, not all attempts to degrade pattern goodness have eliminated a global advantage. In particular, two studies of the effect of sparsity on global precedence have shown conflicting results (Martin, 1979; Navon, 1983). Martin (1979) found that patterns made of few local elements generated a local advantage in RT, whereas Navon (1983) found that few-element patterns, as well as many-element patterns, showed the typical global advantage.

Consider some differences in stimuli and task instruction that might explain the robustness of the global advantage in Navon's study and its vulnerability to sparsity in Martin's study. The stimulus patterns used in the Martin (1979) and Navon (1983) studies were different in kind and in size. Navon's geometric shapes were about half the size of Martin's letters. One explanation for the disparate findings is that a global letter is more easily degraded than is a global geometric form. There is no a priori reason to expect processing differences between letters and other shapes in this paradigm, but it is possible that features of the square-diamond shapes (e.g., 
closure) help preserve the coherence of the form even when they are sparse. A better explanation is that sparsity has less effect on perceived pattern goodness in smaller patterns than in larger ones. That is, removing local elements from a global form subtending $2.0^{\circ}$ (Navon) degrades the quality of form less than if the global form subtended $4.05^{\circ}$ (Martin). Only in the Martin study were ratings of the relative recognizability of the manyelement and sparse forms obtained to verify that sparsity actually compromised good form. Another possibility is that the local elements of Navon's forms were simply too small to discriminate easily.

The two studies differed in how subjects were instructed to focus their attention and what they had to judge. In the Martin (1979) study, subjects identified either the global or local form in separate trial blocks (the original selectiveattention, identification task used by Navon, 1977). In the Navon (1983) study, subjects had a divided attention task in which they judged whether two patterns were the same or different (based on both levels). The measure of global precedence was the RT advantage when the difference was at the global level relative to when the difference was at the local level. Navon favored the divided-attention, same-different task because he postulated that a task that did not restrict the attentional focus of the subject ${ }^{1}$ or require recognition of the form was more likely to elicit a global advantage. It is possible, then, that the effect of sparsity on the global advantage was attenuated by the task conditions used by Navon.

\section{EXPERIMENT 1A}

In Experiments 1A and 2, the effects of pattern goodness on global precedence were reexamined under varied attentional and task conditions. The first part of Experiment $1 \mathrm{~A}$ used task conditions and stimulus forms that apparently facilitated a global advantage in the Navon (1983) study. As in the Navon study, subjects judged whether two patterns were the same or different on both the global and local levels (divided attention task), whether the stimulus patterns were compound forms made of squares and diamonds, and whether the goodness of the global form was altered by varying the number of local elements constituting the configuration. Unlike in the Navon study, the patterns were twice as large and were similar to those in the Martin (1979) study, and the pattern goodness of the global and local shapes were rated by the subjects. Navon's suggestion that conscious attentional processes might affect global precedence was tested in the second part of Experiment $1 \mathrm{~A}$ by instructing the subjects to base their same-different judgments on either the global or the local level. Experiment 2 tested whether or not the task instruction to identify the pattern would enhance the effects of pattern goodness on global precedence and lead to a local precedence effect as shown in the Martin study.

If global precedence is not easily perturbed by methodological nuances, then all variations of attentional and task instructions should generate similar results. Two pre- dictions follow from the Gestalt perspective. If a global precedence effect depends on the quality of the global configuration, then patterns having global forms rated high in perceived goodness should produce reliable global precedence effects. Those rated low should not.

\section{Method}

Subjects. Eight adult subjects participated, ranging in age from 24 to 44 years. All reported normal or corrected-to-normal vision and were paid $\$ 5$ per hour for their participation.

Apparatus. The stimuli were presented on the monitor of a Macintosh SE computer. The mean luminance of the screen was $20 \mathrm{~cd} / \mathrm{m}^{2}$. The Michelson contrast [(max luminance $-\min$ luminance $) /(\max$ luminance $+\min$ luminance $)]$ was .94 . The computer also controlled the randomization and presentation of stimuli together with the timing and recording of responses. The subjects viewed the display at a distance of $60 \mathrm{~cm}$. Two computer keys (" $x$ " and "..") were dedicated to recording the subjects' responses. Four of the subjects used the index finger of their dominant hand to report same; four used their nondominant hand for same. In the goodness rating tasks, all subjects used the right key ("“.") to choose the display on the right and the left key (" $x$ ") to choose the display on the left.

Stimuli. The stimulus patterns were constructed and labeled in accordance with Experiment 2 of the Navon (1983) study. The patterns were compound forms consisting of global geometric shapes made of local shapes. The figures were black on a white background. The three stimulus sets shown in Figure 1 differed only in the size of the global form and in the number and spacing of the local elements. Each set contained all combinations of squares and diamonds. The global shape in the many-element set consisted of eight local elements. The global shape in the sparse set was equal in size to the many-element set but had only four local elements. The global shape in the dense set contained four local elements, but it was $74 \%$ as large as the global patterns of the many-element and sparse sets. The distance between midpoints of adjacent elements was $1.5 \mathrm{~cm}$ $\left(1.44^{\circ}\right)$ in the many-element set, $3.0 \mathrm{~cm}\left(2.86^{\circ}\right)$ in the sparse set, and $2.0 \mathrm{~cm}\left(1.91^{\circ}\right)$ in the dense set. Actual interelement distance depended on the specific configuration of squares and diamonds. For example, in the large square made of small squares, interelement spacing was $.70 \mathrm{~cm}\left(.67^{\circ}\right)$ in the many-element set, $2.20 \mathrm{~cm}$ $\left(2.10^{\circ}\right)$ in the sparse set, and $1.20 \mathrm{~cm}\left(1.14^{\circ}\right)$ in the dense set.

The height of the global square was $3.8 \mathrm{~cm}\left(3.62^{\circ}\right)$ in the manyelement and sparse sets and $2.8 \mathrm{~cm}\left(2.67^{\circ}\right)$ in the dense set. The height of the global diamond was $4.5 \mathrm{~cm}\left(4.29^{\circ}\right)$ in the manyelement and sparse sets and $3.3 \mathrm{~cm}\left(3.14^{\circ}\right)$ in the dense set. The local square in all sets was $0.8 \mathrm{~cm}\left(0.76^{\circ}\right)$ wide and high. The local diamond in all sets was $1.0 \mathrm{~cm}\left(0.95^{\circ}\right)$ wide and high. A pair of figures was presented side by side $4 \mathrm{~cm}$ apart in the center of the monitor. The width of the display subtended $11.5^{\circ}$ for the manyelement and sparse patterns and $9.4^{\circ}$ for the dense patterns.

Design. For each stimulus set (many-element, sparse, and dense), the subjects judged whether a pair of figures was the same or different under two conditions: a divided-attention and a selectiveattention task. In the divided-attention task, same was the correct judgment only if the two displays were identical (i.e., each display was a large square made of small diamonds). Differences were correctly judged if the two displays differed on the global level, the local level, or on both the global and local levels. In the selective attention procedure, the subjects were told to base their judgment of same or different on either the global or local level, depending on the instructions. The shapes on the irrelevant level were always the same across the stimulus pair.

The divided-attention task for each stimulus set contained 360 trials ( 120 for each stimulus set). On $50 \%$ of the trials, the two patterns were identical (same). Of the remaining trials, 16 pairs 
Local Square Local Diamond Many-element

Global square
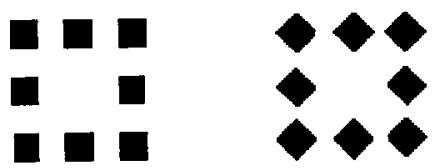

Global diamond
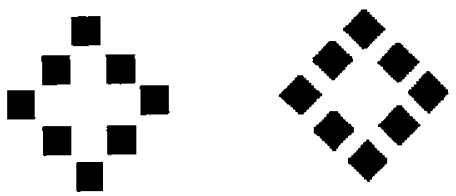

Sparse

Global square

Global diamond

I

$\square$

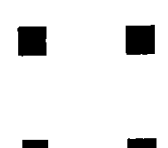

西
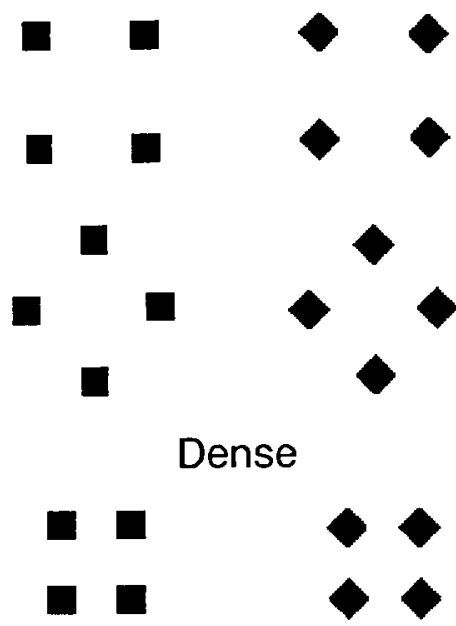

Dense

Global square

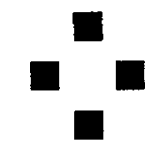

Global diamond

1. Stimulus sets for square and diamond patterns.

differed on the global level, 16 differed on the local level, and 16 differed on both global and local levels. The selective-attention task had 240 trials ( 80 for each stimulus set). The 80 selective trials for each stimulus set were presented in four blocks in which the level (global or local) and the form on the irrelevant level (square or diamond) were held constant in each trial block. For each block, $50 \%$ of the trials had identical patterns (same). The stimulus pairs on the remaining trials had either global or local differences.

In addition to the two RT tasks, the subjects were also asked to rate the goodness of the forms in a forced-choice task. In a trial, the subjects were shown two of a given display from different stimulus sets (e.g., a large square made of small diamonds from the many-element set and the sparse set) and asked to select in which display it was easier to recognize the form. Good form should facilitate recognition of the pattern (Robertson, 1986). The subjects made the judgment on the global forms and on the local forms in separate trial blocks. Each trial block contained 72 trials.

Procedure. Each subject performed all tasks in three sessions, each approximately $45 \mathrm{~min}$ long. One stimulus set was used in each session. Thus, the subjects performed one divided-attention and four selective-attention tasks per session. The four selective-attention tasks for each stimulus set were always given consecutively. One of the two goodness-rating tasks (global or local) was performed last in the first session, and the other was performed last in the second session. The presentation order of the stimulus sets, the goodness-rating tasks, and the global and local block of each selective-attention task was balanced across subjects. Also for each subject, the order of the divided- and selective-attention tasks and the global-local blocks within each selective attention task alternated across sessions. In all tasks, each pattern appeared equally as the right and left member of the stimulus pair.

Each task was preceded by a practice run in which the subject saw at least one instance of each figure used in that task. The dividedattention task had 10 practice trials, the selective-attention task had 8 practice trials, and the goodness-rating task had 12 practice trials. Feedback for accuracy and speed was printed on the screen after each trial in the practice runs to help the subject get oriented to the specific task directions.

For the selective- and divided-attention tasks, each trial began with a l-sec display of a fixation point (a small star) in the center of the screen, followed by the stimulus display. The subject's response turned off the stimulus. The intertrial interval between the subject's response and stimulus onset was $2.5 \mathrm{sec}$. The subjects were urged to respond as fast as possible without making errors. In the goodness-rating tasks, the trial began with a word describing the shape to be judged on that trial (e.g., "square" or "diamond") centered near the top of the screen for $1 \mathrm{sec}$. The fixation point and stimulus occurred as before.

\section{Results and Discussion}

Goodness ratings. The global form of the manyelement pattern was judged to be the easiest to recognize. It was judged as easier $88 \%$ of the time relative to sparse patterns and $83 \%$ of the time relative to dense patterns. These values exceeded chance in both instances $[t(7)=$ $3.0, p<.03$, and $t(7)=2.51, p<.05$, respectively] In contrast, density had little effect on perceived goodness of the global form. The subjects chose dense patterns over sparse patterns on $57 \%$ of the trials $(t<1)$.

The number and spacing of the local elements had little effect on the perceived goodness of the local form. The subjects judged sparse patterns as easier to recognize than many-element patterns on $68 \%$ of the trials, dense patterns as easier to recognize than many-element patterns on $59 \%$ of the trials, and sparse patterns as easier to recognize than dense patterns $62 \%$ of the time. None of these percentages significantly exceeded chance $[t(7)<$ $1.37, p>.10$, in each instance].

Divided-attention task. Error rates shown in Table 1 were low (mean $=2.8 \%$ ). The results of an analysis of variance (ANOVA) on individual mean error rates [subjects $\times$ stimulus set $\times$ type of trial (same trials and those with global differences, local differences, or both)] showed no systematic pattern among the four types of trials $[F(3,21)=1.22, p=.33]$, the stimulus sets $(F<1)$, or their interaction $(F<1)$. A speed-accuracy tradeoff was not indicated. Incorrect responses were excluded from further analyses.

Table 1 shows the mean RTs in each stimulus set for the same trials and global differences, local differences, or differences at both levels. Note that many-element pat- 
Table 1

Mean Response Times (RTs, in Milliseconds) for Same and Different Trials by Stimulus Set in the Divided-Attention Task

\begin{tabular}{|c|c|c|c|c|c|c|c|c|c|c|c|c|}
\hline \multirow{4}{*}{$\begin{array}{c}\text { Stimulus } \\
\text { Set } \\
\end{array}$} & \multicolumn{8}{|c|}{ Type of Trial } & & & & \\
\hline & & & \multicolumn{6}{|c|}{ Different } & \multicolumn{4}{|c|}{ Comparisons } \\
\hline & \multicolumn{2}{|c|}{ Same } & \multicolumn{2}{|c|}{ Local } & \multicolumn{2}{|c|}{ Global } & \multicolumn{2}{|c|}{$\begin{array}{c}\text { Local \& } \\
\text { Global }\end{array}$} & \multicolumn{2}{|c|}{$\begin{array}{c}\text { Global } \\
\text { Advantage* }\end{array}$} & \multicolumn{2}{|c|}{$\begin{array}{c}\text { Redundancy } \\
\text { Gain } \dagger\end{array}$} \\
\hline & $M$ & ER & $M$ & ER & $M$ & ER & $M$ & ER & $M$ & $S E$ & $M$ & $S E$ \\
\hline Many & 676 & $2.1 \%$ & 743 & $6.2 \%$ & 632 & $1.9 \%$ & 592 & $1.3 \%$ & $111 \ddagger$ & 43 & $40 \S$ & 8 \\
\hline Sparse & 645 & $3.1 \%$ & 726 & $2.5 \%$ & 688 & $2.5 \%$ & 655 & $3.1 \%$ & 38 & 28 & 33 & 26 \\
\hline Dense & 563 & $2.2 \%$ & 620 & $3.1 \%$ & 608 & $3.1 \%$ & 574 & $1.9 \%$ & 12 & 19 & 34 & 18 \\
\hline
\end{tabular}

Note-ER = error rate. ${ }^{*}$ Local different RT minus global different RT. †Global different RT minus local + global different RT $\ddagger p<.05 . \quad \$ p<.01$.

terns showed a large global advantage (difference $=$ $111 \mathrm{msec}$ ), whereas the global advantage was relatively smaller in the sparse (difference $=38 \mathrm{msec}$ ) and dense (difference $=12 \mathrm{msec}$ ) sets. Furthermore, RTs for trials with differences on both levels were faster than RTs for trials with global differences (mean difference $=35 \mathrm{msec}$ ), suggesting the possibility of interlevel facilitation or redundancy gain.

An ANOVA was performed on the individual mean RTs [subjects $\times$ stimulus set $\times$ type of trial (same trials and those with global differences, local differences, or both)]. The critical interaction between stimulus set and the type of trial was reliable $[F(6,42)=2.74, p<.03]$. Planned contrasts assessed the global advantage within each stimulus set. A reliable global advantage only occurred in the many-element set $[F(1,7)=6.76, p<.04]$. The globallocal differences in the sparse and dense patterns were not reliable $[F(1,7)=1.90, p=.21$, and $F<1$, respectively]. The main effects for the type of trial and stimulus set did not reach significance $[F(3,21)=3.04, p<$ .06 , and $F(2,14)=1.67, p=.22$, respectively].

Although the present data were derived from a dividedattention, same-different task similar to Navon's (1983) method, the results more closely resemble Martin's (1979) findings, in which the size of a global advantage is influenced by the number of local elements constituting the global configuration. Moreover, the perceived goodness of the global form (as rated by the subjects) reflected the sparsity manipulation and predicted the occurrence of a global advantage.

Redundancy gains. If global information is encoded and available before local information, as suggested by Navon (1977), then patterns with both global and local differences and those with only global differences should generate equivalent RTs. However, if local differences improve processing beyond that attained when only the global source is available (redundancy gain), then a strict global-to-local processing order is ruled out (Miller, 1981). As seen in Table 1, the redundancy gains derived from the three stimulus sets were similar in magnitude (average $=35 \mathrm{msec}$ ). However, when each stimulus set was tested separately, the value was only reliable in the many-element set $[F(1,7)=23.33, p<.01]$, not in the sparse and dense sets $[F(1,7)=1.49, p=.26$, and $F(1,7)$ $=3.35, p=.11$, respectively]. At the least, these data show that local information is occasionally encoding faster than global information. ${ }^{2}$ The statistical results, however, suggest that fast encoding of local information is only found in those patterns that generated a global advantage, which is not consistent with Navon's early encoding hypothesis.

Interference effects. If a global precedence effect results from earlier encoding of the global versus the local form, then global information inconsistent with local information could delay or interfere with local judgments, but not vice versa. Two types of trials-same trials and trials with differences on both levels-allowed for a comparison between consistent (e.g., a large square made of small squares) and inconsistent patterns (e.g., a large square made of small diamonds). Since the same-different task required the subjects to compare shapes between the stimulus pair rather than within a single pattern, the consistency of form within a single stimulus is irrelevant to the task but difficult to ignore. If global information is encoded first, a distinct pattern of interference should occur in trials with differences on both levels. Many-element patterns that show a global advantage should yield similar RTs for consistent and inconsistent judgments because detecting the global difference is sufficient to make the correct response. Conversely, few-element patterns in which no global advantage is observed should show longer RTs for inconsistent patterns because contradictory information interferes with whichever level is evaluated first. A similar but weaker prediction can be made for same trials. In many-element patterns, global forms are likely to be compared before local forms. In this case, only the local comparison is subject to interference (by conflicting global information). In few-element patterns, the comparisons may be performed in any order, and either form is likely to sustain interference by conflicting information on the other level. Thus, for same trials, manyelement patterns are likely to show less interference than are few-element patterns.

An ANOVA of the individual mean RTs [subjects $x$ stimulus set $\times$ type of trial (same trials and trials with differences on both levels) $\times$ consistency] tested these predictions. On average, RT was $29 \mathrm{msec}$ longer for inconsistent patterns than for consistent patterns $[F(1,7)=$ $9.33, p<.02]$. All three stimulus sets showed this effect, and there was no reliable interaction between the consistency factor and either stimulus set or type of trial $(F<1)$ in each instance. Thus, the magnitude of the in- 
Table 2

Mean Response Times (RTs, in Milliseconds) for Same and Different Trials by Stimulus Set in the Selective-Attention Task

\begin{tabular}{|c|c|c|c|c|c|c|c|c|c|c|c|c|}
\hline \multirow{3}{*}{$\begin{array}{c}\text { Stimulus } \\
\text { Set }\end{array}$} & \multicolumn{6}{|c|}{ Same } & \multicolumn{6}{|c|}{ Different } \\
\hline & \multicolumn{2}{|c|}{ Local } & \multicolumn{2}{|c|}{ Global } & \multicolumn{2}{|c|}{$\begin{array}{c}\text { Global } \\
\text { Advantage* }\end{array}$} & \multicolumn{2}{|c|}{ Local } & \multicolumn{2}{|c|}{ Global } & \multicolumn{2}{|c|}{$\begin{array}{c}\text { Global } \\
\text { Advantage* }\end{array}$} \\
\hline & $M$ & ER & $M$ & ER & $M$ & $S E$ & $M$ & ER & $M$ & ER & $M$ & $S E$ \\
\hline Many & 698 & $5.6 \%$ & 598 & $3.8 \%$ & $100 \dagger$ & 9 & 736 & $4.4 \%$ & 616 & $3.8 \%$ & $120 \dagger$ & 24 \\
\hline Sparse & 610 & $3.8 \%$ & 623 & $3.8 \%$ & -11 & 63 & 663 & $6.3 \%$ & 644 & $3.1 \%$ & 19 & 43 \\
\hline Dense & 613 & $4.4 \%$ & 596 & $3.8 \%$ & 17 & 17 & 653 & $1.9 \%$ & 662 & $2.5 \%$ & -9 & 43 \\
\hline
\end{tabular}

Note-ER = error rate. ${ }^{*}$ Local RT minus global RT. $\quad \nmid p<.001$.

terference effects was not predicted by the global advantage in RT. Instead, interlevel interference occurred in both many-element and few-element forms, suggesting that encoding differences between global and local information as specified by interlevel interference might not account for the global advantage shown in many-element patterns.

Selective-attention task. In this task, global and local RTs and error rates could be compared on same trials as well as on different trials. Error rates (mean $=3.9 \%$ ) are shown in Table 2. The results of an ANOVA of individual mean error rates [subjects $\times$ stimulus set $\times$ type of trial (same vs. different) $\times$ level (global vs. local)] showed no reliable differences between same and different trials $(F<1)$, among the stimulus sets $[F(2,14)=1.16, p=$ $.34]$, or between global and local responses $(F<1)$. All interactions between the factors were also nonsignificant [ $F<1$ for each two-way interaction, and $F(2,14)=$ $2.40, p=.13$, for the three-way interaction]. As a speed-accuracy tradeoff was not indicated, incorrect responses were excluded from further analyses.

The RT data shown in Table 2 essentially replicate the findings from the divided-attention task. In both same and different trials, the global advantage in RT was larger in the many-element set than in the few-element sets. An ANOVA of individual mean RTs [subjects $\times$ stimulus set $\times$ type of trial (same vs. different) $\times$ level (global vs. local)] showed that the critical interaction of stimulus set and level was reliable $[F(2,14)=4.44, p<.04]$, with no interaction with type of trial $(F<1)$. Further planned contrasts indicated that the difference between global and local RT was reliable in the many-element set $[F(1,7)=$ $57.03, p<.001]$ but not in the sparse or dense set ( $F<1$ in each instance). The reported significance levels are adequate when adjusted for multiple comparisons (modified Bonferroni test).

If global precedence results at least in part from attentional processes, then a task that constrains attention to either the global or local level might reduce the magnitude of the global advantage (Navon \& Norman, 1983). An analysis of both tasks together [subjects $\times$ stimulus set $\times$ type of trial (local vs. global) $\times$ task (divided vs. selective attention)] showed the expected interaction between stimulus set and the type of trial $[F(2,14)=8.60$, $p<.01]$, with a reliable global-local difference in the many-element set $[F(1,7)=13.76, p<.01]$ but not in the sparse or dense sets $(F<1$ in both instances). The significance levels were sufficient when adjusted for the multiple follow-up tests (modified Bonferroni test). The interesting finding is that there was no significant main effect for the attention factor $(F<1)$ for any interaction between attention and stimulus set $[F(2,14)<2.61, p>$ $.10]$, or for the type of trial $(F<1)$. Restricting attention by task directions did not affect either the overall response latency or the occurrence or magnitude of the global advantage.

These results provide further support for the view that a global precedence effect depends on the quality of the global form. Reducing the number of elements lowered perceived ratings of goodness and eliminated a global advantage. Furthermore, these data show that the processing advantage for global information in many-element forms was not easily disrupted by conscious attentional strategies. In the selective-attention task, the subjects had only to judge information on one level with no distracting variation on the irrelevant level. Yet a global advantage still occurred in many-element patterns, suggesting that global precedence arises from a processing bias toward the pattern over its parts, providing that the pattern is well-structured. Moreover, although good form, as well as postperceptual processes, might enhance encoding, evidence of interlevel interference in both "good" and "poor" patterns suggests that global and local information was encoded and available within a similar time course in all patterns. As a consequence, the effects of good form are reasonably ascribed to a postperceptual process.

Although the stimuli and method in the present study (particularly the divided-attention task) were based on the Navon (1983) study, the results failed to replicate Navon's findings, in which a global precedence effect occurred in both few- and many-element patterns. One difference between the Navon study and the present one is the size of the global and local forms. Navon's patterns were half as large as those in the present study. The next experiment examined whether sparsity reduces global precedence only in large patterns (replication of Experiment 1A) but not in small ones (replication of the Navon study).

\section{EXPERIMENT 1B}

The primary objective of this experiment is to determine if the discrepant findings between Experiment $1 \mathrm{~A}$ and the Navon (1983) study can be attributed to the size of the patterns. If so, then we must consider how this oc- 
curs. One possibility is that the sparsity manipulation may have less effect on perceived pattern goodness in small patterns. Another possibility is that the small size of Navon's local shapes may have contributed to their slower processing and obscured the effects of sparsity. Although local elements in Experiment 1 A subtended $0.8^{\circ}$, Navon's local elements were $0.23^{\circ}$, a size approaching acuity limits (Navon, 1983; Navon \& Norman, 1983).

To test these hypotheses, the divided-attention task used in Experiment $1 \mathrm{~A}$ was repeated both with the same patterns and with patterns approximately half as large. Rather than rating good form by the forced-choice procedure of Experiment 1A, the subjects rated the goodness of form of large and small patterns individually on a 5-point rating scale. This procedure avoided a direct comparison between large and small patterns, which could have favored the more salient large forms.

\section{Method}

Subjects. Eighteen subjects participated in the divided-attention task and 12 subjects participated in the rating task. Six subjects participated in both tasks in separate sessions 3 weeks apart. In these cases, the divided-attention task was performed in Session 1. The age of the subjects ranged from 18 to 35 years. All reported normal or corrected-to-normal vision and were offered course credit for their participation.

Stimuli. The apparatus and set-up were identical to those in Experiment 1A. The large stimulus forms were also the same as in Experiment 1A. The small stimulus forms were identical to the large patterns except that they were approximately half as large. The size parameters for the small patterns were as follows: The height of the global square was $2.0 \mathrm{~cm}\left(1.91^{\circ}\right)$ in the many-element and sparse sets and $1.5 \mathrm{~cm}\left(1.43^{\circ}\right)$ in the dense set. The height of the global diamond was $2.5 \mathrm{~cm}\left(2.39^{\circ}\right)$ in the many-element and sparse sets and $1.75 \mathrm{~cm}\left(1.67^{\circ}\right)$ in the dense set. In all sets, the local square was $0.4 \mathrm{~cm}\left(0.38^{\circ}\right)$ wide and high, and the local diamond was $0.5 \mathrm{~cm}\left(0.48^{\circ}\right)$ wide and high. In the divided-attention task, the small patterns were presented $2.2 \mathrm{~cm}$ apart in the center of the monitor. The width of these displays was $5.90^{\circ}$ for many-element and sparse patterns and $4.95^{\circ}$ for dense patterns.

Design. The same divided-attention task used in Experiment IA was employed. Recall that many-element, sparse, and dense patterns were presented in separate blocks. Thus, there were six blocks of trials in this experiment (two sizes $x$ three stimulus sets).

Unlike in the forced-choice rating procedure of the previous experiment, a single pattern was presented on each trial. The subjects were required to rate the goodness or recognizability of the global or local shape by using a 5-point scale. The subjects registered their response by depressing the appropriate key labeled 1 through 5 on the computer keyboard. A rating of 1 indicated that the form was very hard to recognize, whereas a rating of 5 indicated that the form was very easy to recognize. Separate blocks were run for global and local forms, but large and small patterns were randomly presented within the same block. This permitted a comparison of ratings between sizes without simultaneous presentation, which could have favored the larger forms. Each block contained 96 trials. The first 24 trials were considered practice and were excluded. The dependent measure was the mean rating of patterns of each stimulus set and size.

Procedure. The divided-attention task (six blocks) was performed in a 1-h session. During the first section of the session, half of the subjects saw the small patterns, and half saw the large patterns. The alternate size patterns were shown in the second section. In each section, the six possible orderings of three stimulus sets were balanced across subjects. For each subject, the order of presenta- tion in the second section was the reversal of that in the first section. This counterbalancing scheme generated six orders across the size and stimulus-set factors.

The rating task was completed in one 30 -min session. Half of the subjects performed global ratings first; the other half performed local ratings first. For the subjects performing both procedures, the two sessions were separated by approximately 3 weeks, with the divided-attention task presented first. Since half of the subjects in the rating task had never seen these compound patterns while half had participated in the divided-attention task, the effects of prior experience with the stimuli could be examined.

\section{Results and Discussion}

Divided-attention task. The analyses of these data directly test the hypothesis that the size of the pattern mediates the effects of sparsity on global precedence. The global advantage (local minus global) in error rates and RTs was calculated separately for large and small patterns in the many- and few-element (average of sparse and dense) format. These data are presented in Table 3.

The error values presented in Table 3 show a small global advantage in accuracy $(2.8 \%)$. An ANOVA of the individual mean global advantage in error rates (subjects $X$ stimulus format $\times$ size) showed no reliable effect for stimulus format $[F(1,17)=2.40, p=.14]$, size $(F<1)$, or their interaction $(F<1)$. There was no evidence for a speed-accuracy tradeoff, and incorrect trials were excluded from further analysis.

As shown in Table 3, the magnitude of the global advantage in RT was similar in the many-element versions of both large and small patterns and in the few-element versions of the small forms. The few-element, large patterns showed a small local advantage. The analysis of these data included the between-subject factor for order (six levels) to determine whether the order of presentation might interact with the sparsity and size manipulations. An ANOVA on the individual mean global advantage in RT (subjects $\times$ order $\times$ stimulus format $\times$ size) confirmed a reliable interaction between stimulus format and size $[F(1,12)=7.00, p<.03]$. Planned comparisons showed that for many-element patterns, the global advantage did not differ between the large and small versions $(F<1)$, whereas for few-element patterns, the local-global difference was larger in small patterns than in large patterns $[F(1,17)=3.11, p<.01]$. Reported significance levels were adequate when adjusted by the modified Bonferroni procedures. These findings support the hypothesis that sparsity reduces global precedence if the patterns are as large as those used in Experiment $1 \mathrm{~A}$

Table 3

Mean Global Advantage* (in Milliseconds) by Stimulus Format and Size

\begin{tabular}{cccccc}
\hline & \multicolumn{4}{c}{ Size of Patterns } \\
\cline { 2 - 5 } Stimulus Set & \multicolumn{2}{c}{ Large } & & \multicolumn{2}{c}{ Small } \\
\cline { 2 - 4 } \cline { 5 - 6 } & $M$ & ER & & $M$ & ER \\
\hline Many-element & 85 & $.9 \%$ & & 99 & $5.2 \%$ \\
Few-element & -15 & $2.3 \%$ & & 73 & $2.8 \%$ \\
\hline
\end{tabular}

Note-ER $=$ error rates. $\quad$ *Local RT minus global RT. 
but not if they are as small as those used by Navon (1983). In addition, reliable main effects were found for size $[F(1,12)=6.20, p<.03]$ and stimulus format $[F(1,12)$ $=13.46, p<.01]$, but these are best considered in light of the interaction.

The main effect for order was reliable $[F(5,12)=3.82$, $p<.03$ ], but the pattern among the six orderings was not systematic. The nonsignificant interaction between order and size $(F<1)$, stimulus format $[F(5,12)=$ $1.056, p=.43]$, or their interaction $[F(5,12)=2.95$, $p=.06$ ] suggested that order did not mediate the association between the size of the patterns and the effects of sparsity.

Goodness ratings. Table 4 shows the mean rating for global and local forms in each stimulus set for large and small patterns. The mean global ratings were similar in magnitude for large and small patterns (means $=3.45$ and 3.46 , respectively), whereas mean local ratings were higher for larger patterns (mean $=3.87$ ) than for smaller ones (mean $=3.05$ ). Both large and small patterns show similar effects of sparsity on perceived pattern goodness. In both cases, global ratings were higher for many-element patterns than for sparse or dense patterns, whereas local ratings showed relatively little change across stimulus sets.

The analysis of these data also contained two betweensubject factors, order and experience. Recall that half of the subjects performed the global ratings first and half performed the local ratings first. Furthermore, half of the subjects in each ordering had participated in the RT task, whereas the others had no prior experience with the compound patterns used in this study. An analysis of the individual mean ratings [subjects $x$ order $x$ experience $x$ type of trial (global vs. local) $\times$ size $\times$ stimulus set] yielded only four reliable effects. The observation that only local ratings were affected by the size of the pattern was confirmed by the reliable interaction of size and type of trial $[F(1,8)=7.86, p<.01]$. Global ratings were very similar for the large and small patterns (means $=$ 3.45 and 3.46 , respectively), whereas local ratings were higher for large patterns (mean $=3.87$ ) than for small ones (mean $=3.05$ ). The hypothesis that the size of the patterns affects local ratings more than global ratings was confirmed $[F(1,11)=8.47, p<.02]$. In sum, small local forms were harder to recognize than were large local forms, whereas small and large global forms appear to be equally recognizable.

Table 4

Mean Ratings of Good Form for Global and Lacal Patterns by Stimulus Set and Stimulus Size

\begin{tabular}{|c|c|c|c|c|}
\hline \multirow[b]{3}{*}{ Stimulus Set } & \multicolumn{4}{|c|}{ Type of Trial } \\
\hline & \multicolumn{2}{|c|}{ Global } & \multicolumn{2}{|c|}{ Local } \\
\hline & Large & Small & Large & Small \\
\hline Many & 3.97 & 3.92 & 3.94 & $3 . \overline{16}$ \\
\hline Sparse & 3.11 & 3.26 & 3.76 & 2.94 \\
\hline Dense & 3.29 & 3.21 & 3.91 & 3.05 \\
\hline Mean & 3.45 & 3.46 & 3.87 & 3.05 \\
\hline
\end{tabular}

The effect of sparsity on global-local ratings was shown by the reliable interaction of stimulus set and type of trial $[F(2,16)=7.86, p<.01]$. Simple effects tests showed that for global ratings, many-element patterns were judged higher in pattern goodness than were sparse patterns $[F(1,16)=19.35, p<.001]$ or dense patterns $[F(1,16)$ $=16.15, p<.001]$. For local ratings, there were no reliable differences among the stimulus sets $(F<1)$. These significance levels were adequate when adjusted by modified Bonferroni procedures. Furthermore, the nonsignificant interaction of size, stimulus set, and type of trial $[F(2,16)=1.29, p=.30]$ supports the observation that large and small patterns showed similar effects of sparsity. The important finding is that sparsity reduces pattern goodness in configurations as large as those used in $\mathrm{Ex}$ periment 1A and as small as those used by the Navon (1983) study.

In addition to these interactions, there were also reliable main effects for size $[F(1,8)=6.89, p<.04]$ and stimulus set $[F(2,16)=12.69, p<.001]$, but these effects appeared to be by-products of the previous interactions. Furthermore, there was no evidence in these data that prior experience with the patterns or order of presentation affected the subjects' ratings. All other main and interaction effects were nonsignificant $[F(1,16)<2.62$, $p>.11$, for effects involving stimulus set, and $F(1,16)$ $<3.10, p>.12$, for all others].

In sum, these data show that the size of the stimuli may determine whether sparsity reduces global precedence. Sparsity reduced the observed global advantage in patterns subtending approximately $4^{\circ}$ (Experiment 1A) but not in patterns half as large (similar to the Navon study). However, the maintenance of a global advantage in small, few-element patterns does not reflect a preservation of pattern goodness under the sparsity manipulation. Sparsity reduced global ratings in both large and small patterns. Rather, the rating data suggested that small patterns have local elements that are difficult to discriminate and hard to process. Thus, small configurations may not provide a strong test of the effects of sparsity on global precedence because the relative discriminability of the global and local forms may be too strongly biased toward the global forms.

\section{EXPERIMENT 2}

The previous experiments examined some factors that might mediate the effects of sparsity on global precedence. Although task-directed attentional constraints did not interact with the sparsity manipulation, sparsity only reduced the global advantage when patterns were as large as those used by Martin (1979) and not when patterns were as small as those used by Navon (1983). A third factor that could influence how sparsity affects global precedence is the processing requirements of the task itself.

Navon (1983) had suggested that global precedence is less vulnerable to the effects of sparsity if it is derived from a task that did not require form recognition (e.g., 
same-different task). A strong interpretation of this claim is ruled out by the results of the same-different task in Experiment $1 \mathrm{~A}$, in which the global advantage was reduced and unreliable in few-element patterns. Although the present results are more consistent with the Martin (1979) study than with the Navon (1983) study, they do not replicate the local precedence effect elicited by Martin's sparse forms. Navon's conjecture provides an explanation. If good form is even more important to global precedence when subjects must identify (hence recognize) the shape, as they did in the Martin study, then "poor" global forms would be expected to produce a local precedence effect. In Experiment 2, this hypothesis was tested by presenting to subjects a single pattern on each trial and asking them to identify either the global or local form in separate trial blocks. Unlike in Experiment 1A but in accord with the Martin study, the forms on the irrelevant level varied across trials, providing an opportunity to examine interlevel interference.

\section{Method}

Subjects. Eight adult subjects participated. Five had also participated in Experiment 1A. ${ }^{3}$ The age range was 24 to 44 years. All reported normal or corrected-to-normal vision. The subjects were paid $\$ 5$ per hour for their participation.

Stimuli. The apparatus and set-up were identical to those in the previous experiment. The stimulus forms were the same as in Experiment $1 \mathrm{~A}$. However, only a single display was presented on each trial.

Design. A selective-attention, identification task was used in which the subjects were told to identify the shape at either the global or local level, depending on the specific instructions for the condition. There were two choices: "square" or "diamond." Each combination of global and local form appeared equally often so that the shapes on the irrelevant level were not correlated with the shapes on the relevant level or held constant. The global and local shapes in a given display were either the same (consistent) or different (inconsistent), providing an assessment of interference from conflicting information on the irrelevant level. There were separate trial blocks for each stimulus set (many, sparse, and dense) and type of judgment (global vs. local). Each block consisted of $\mathbf{4 0}$ trials, allowing 10 repetitions of each combination of the two shapes. The total number of trials in the experiment was 240 .
The $\mathrm{S}$ and $\mathrm{D}$ keys always recorded square or diamond decisions, respectively. The subjects placed both index fingers on the designated keys, pressing the key appropriate to their decision. The mapping of the response keys to square and diamond decisions was held constant across tasks and subjects, since data from both shapes were analyzed together.

Procedure. Each subject performed all conditions in one 45-min session. The order of presentation of the stimulus sets (manyelement, sparse, or dense) and type of response (global or local) was counterbalanced within and across subjects.

For each trial block, the subjects were told to base all decisions on either the global or local level. A practice session of eight trials preceded each block in which the subject saw two examples of each pattern and received accuracy and RT feedback after each trial. Each trial began with a fixation point (a small star) in the center of the monitor for $1 \mathrm{sec}$. The stimulus pattern followed immediately and was terminated by the subject's response. The intertrial interval was $2.5 \mathrm{sec}$. The subjects were urged to respond as quickly as possible without making errors.

\section{Results and Discussion}

Error rates are shown in Table 5. The mean error rate was $5.69 \%$. An ANOVA on individual mean error rates [subject $\times$ stimulus set $\times$ type of trial (global vs. local) $x$ consistency] showed higher errors rates for inconsistent patterns $[F(1,7)=8.99, p<.03]$. Further analysis of the reliable main effect for stimulus set $[F(2,14)=$ $5.52, p<.02]$ showed that sparse patterns had lower errors rates than did either many-element patterns $[F(1,7)=$ $5.9, p<.05]$ or dense patterns $[F(1,7)=12.67, p<$ $.01]$. Error rates did not differ between global and local trials $[F(1,7)=2.43, p=.16]$. More directly related to global precedence, there was no reliable interaction between stimulus set and type of trial $[F(2,14)=1.88, p=$ .19] or between these factors and the consistency factor $[F(2,14)=1.7, p=.23]$. The highest error rate (mean $=$ $\mathbf{8 . 8 \%}$ ) was found for local decisions in the many-element patterns. Since this condition also showed the longest RT (mean $=470 \mathrm{msec}$ ), a speed-accuracy tradeoff was not indicated. Incorrect trials were excluded from further analysis.

Global Advantage. The global and local RTs for correct consistent and inconsistent trials in each stimulus set

Table 5

Mean Global and Local Response Times (RTs, in Milliseconds) by Stimulus Set and Pattern Consistency

\begin{tabular}{|c|c|c|c|c|c|c|c|}
\hline \multirow{3}{*}{$\begin{array}{c}\text { Stimulus } \\
\text { Set }\end{array}$} & \multirow[b]{3}{*}{ Consistency } & \multicolumn{4}{|c|}{ Type of Trial } & \multirow{2}{*}{\multicolumn{2}{|c|}{$\begin{array}{c}\text { Global } \\
\text { Advantage* }\end{array}$}} \\
\hline & & \multicolumn{2}{|c|}{ Local } & \multicolumn{2}{|c|}{ Global } & & \\
\hline & & $M$ & ER & $M$ & ER & $M$ & $S E$ \\
\hline \multirow[t]{3}{*}{ Many } & Inconsistent & 485 & $11.9 \%$ & 433 & $5.0 \%$ & $52 \S$ & 17 \\
\hline & Consistent & 456 & $5.6 \%$ & 405 & $4.4 \%$ & $51 \S$ & 17 \\
\hline & Consistency effect $\dagger$ & 29 & $6.3 \%$ & 28 & $.67 \%$ & & \\
\hline \multirow[t]{3}{*}{ Sparse } & Inconsistent & 435 & $8.1 \%$ & 462 & $4.4 \%$ & -27 & 17 \\
\hline & Consistent & 409 & $2.5 \%$ & 413 & $1.3 \%$ & -4 & 13 \\
\hline & Consistency effect $\dagger$ & 26 & $5.6 \%$ & 49 & $3.1 \%$ & & \\
\hline \multirow[t]{3}{*}{ Dense } & Inconsistent & 435 & $9.4 \%$ & 478 & $8.8 \%$ & $-43 \ddagger$ & 17 \\
\hline & Consistent & 423 & $4.4 \%$ & 431 & $2.5 \%$ & -8 & 19 \\
\hline & Consistency effect $\dagger$ & 12 & $5.0 \%$ & 47 & $6.3 \%$ & & \\
\hline
\end{tabular}

Note-ER = error rates. $\quad{ }^{*}$ Local RT minus global RT. †Inconsistent RT minus consistent RT. $\ddagger p<.05 . \$ p<.02$. 
are shown in Table 5. Similar to the results of Experiment 1A, a global advantage only occurred in manyelement patterns. In addition, sparse and dense patterns showed a small local advantage. An ANOVA on individual mean RTs [subjects $\times$ stimulus set $\times$ type of trial (global vs. local) by consistency] confirmed the interaction between stimulus set and type of trial $[F(2,14)=10.81$, $p<.001]$. Averaged across the consistency factor, the many-element patterns showed a 51-msec global advantage $[F(1,7)=13.98, p<.01]$, whereas the sparse set showed a 16-msec local advantage $[F(1,7)=1.21, p=$ $.31]$ and the dense set showed a 25 -msec local advantage $[F(1,7)=2.50, p=.16]$. Reported significant levels were adequate for multiple comparisons (modified Bonferroni test). The main effects for stimulus set or type of trial were not reliable ( $F<1$ in each case).

As suggested by the Martin (1979) study, the present identification task elicited a global advantage in the "good," many-element patterns and a local advantage in the "poor," few-element patterns. But the local effects were not significant, as they were in the Martin study. Another hint of task-related effects is the relatively small global advantage (mean $=51 \mathrm{msec}$ ) revealed in Experiment 2 compared with its counterpart derived from the divided-attention task (mean $=111 \mathrm{msec}$ ) or the selectiveattention task (mean $=100 \mathrm{msec}$ ) of Experiment $1 \mathrm{~A}$. If the identification process is particularly dependent on good form, then even many-element patterns may suffer because they do not have continuous contours. Although suggestive, these data do not clearly demonstrate that pattern goodness is uniquely important to processes underlying pattern identification. Rather, the most salient finding from these data and those in Experiment $1 \mathrm{~A}$ is that good form predicts global precedence in both tasks.

Interference effects. Strong evidence for the early encoding explanation for global information is the cooccurrence of a global advantage and an asymmetric interference effect favoring global (e.g., a global square interferes with processing a local diamond, but not vice versa). The global-first claim is less supported if a global advantage co-occurs with symmetric interference effects (i.e., conflicting form information on the nonattended level increased both global and local RT) or the occurrence of a global advantage is independent of the interference effects.

In these data, interference is shown by the consistency effect, which is equal to the RT for inconsistent patterns (e.g., large square made of small diamonds) minus RT for consistent patterns (e.g., large square made of small squares). As shown in Table 5, the RT for inconsistent patterns was longer than for consistent ones in each stimulus set, and, on average, the RT to consistent patterns (mean $=423 \mathrm{msec}$ ) was faster than the RT to inconsistent ones [mean $=455 \mathrm{msec} ; F(1,7)=40.81, p<.001$ ] The consistency effect was greater for global responses than for local responses ( $41 \mathrm{msec}$ vs. $22 \mathrm{msec}$ ), the opposite to what would be predicted if global information were interfering with local information, and not vice versa; however, the interaction of type of trial and con- sistency missed significance $[F(1,7)=5.09, p=.06]$. The global and local consistency effects were nearly identical in many-element patterns, whereas global consistency effects were larger than local consistency effects in both sparse and dense patterns; however, the interaction between stimulus set, type of trial, and consistency was not reliable $[F(2,14)=1.25, p=.32]$. The occurrence of a global advantage appeared to be independent of observed consistency effects; all stimulus sets showed consistency effects $(F<1)$, but only the many-element set showed a global advantage in RT.

The co-occurrence of a global advantage and symmetric interference effects in many-element patterns is consistent with models of global precedence that posit only a postperceptual locus (Boer \& Keuss, 1982; Miller, 1981) or both an encoding and a postperceptual basis (Navon \& Norman, 1983; Ward, 1982). ${ }^{4}$ However, similar interference effects were also found in few-element patterns that did not generate a global advantage, suggesting that interference effects may be independent of those processes underlying the global advantage (Lamb \& Robertson, 1989; Robertson et al., 1988).

In sum, the key finding of Experiment 2 was that good form determined global precedence in an identification task as it did in the same-different task of Experiment 1A. Task-related effects such as the tendency toward local precedence in few-element patterns were small and not reliable. An examination of interference effects ruled out a strict global-first explanation for the global advantage but could not determine the encoding and postperceptual processes that might contribute to a global advantage. Clearly, the question of how pattern goodness affects global precedence is not yet answered. One approach to the problem is to combine the manipulation of good form with the manipulation of a second stimulus factor-spatial frequencies-believed to be associated with both Gestalt phenomena (Carrasco, Figueroa, \& Willen, 1986) and global precedence (Navon, 1981). In the next experiment, the effects of sparsity and spatial frequency on global precedence were tested together to explore why a good configuration generates a global advantage.

\section{EXPERIMENT 3A}

Recall that in Navon's explanation, the processing advantage of global over local information results from its relatively larger size or low spatial frequency content. Psychophysical and physiological work has distinguished two classes of visual mechanisms, selectively tuned to low or high spatial frequencies (see Breitmeyer \& Ganz, 1976, or Todd \& Van Gelder, 1979, for a discussion of transient mechanisms; also see Livingstone \& Hubel, 1987, for a discussion of magnocellular pathways). Lowfrequency tuned mechanisms are also known to conduct information faster and to access exogenous attentional processes in the superior colliculus (Breitmeyer \& Ganz, 1976) and the inferior parietal lobe (Bassi \& Lehmkuhle, 1990). Although the fast-conduction feature of these mechanisms implies that earlier encoding of global infor- 
mation is possible, their involvement in attentional control suggests that low frequencies could also attract attention to the global level. It should be noted that the exogenous attentional process posited here may not be under the perceivers' (or experimental task) control (Müller \& Rabbitt, 1989). Thus, there is no inherent conflict between the results of Experiment $1 \mathrm{~A}$ and the claim that low spatial frequencies attract attention to global information.

Three studies have demonstrated that spatial frequency mechanisms are associated with global-local processing. Shulman, Sullivan, Gish, and Sakoda (1986) showed that a decrement in global performance followed adaptation to a low-frequency grating but not to a high-frequency grating. Another study by Shulman and Wilson (1987) found that attention to the global level increased the detectability of a low-frequency grating, suggesting that attention to the spatial frequency band of the global level may mediate the improved performance. Recently Hughes, Fendrich, and Reuter-Lorenz (1990) demonstrated that contrast-balanced stimuli (without spatial frequencies below 3.0 cycles per degree [cpd]) failed to generate global precedence.

The purpose of this experiment is to test the hypothesis that low spatial frequency mechanisms mediate the relationship between good form and global precedence. The identification task of Experiment 2 was used in the current experiment, and the stimuli were adapted from those of the many-element and sparse sets. (The dense set provided little unique information and had smaller global forms than the other two.) The patterns were lowpass filtered with cutoff points at 1.0 and $1.75 \mathrm{cpd}$ (high spatial frequencies were removed) and highpass filtered at 1.0 and $1.75 \mathrm{cpd}$ (low spatial frequencies were removed). The 1.0-to-1.75-cpd boundary range was based on the findings of the Shulman and Wilson (1987) study, in which attending to global patterns as large as the squares and diamonds used in the present study enhanced detection of a 0.5 -cpd sinusoidal grating, whereas attending to the local level enhanced detection of gratings above $2.0 \mathrm{cpd}$. Two cutoff points were used because it was not known beforehand where the most discriminative boundary between low-frequency-tuned and highfrequency-tuned channels might be. Evidence for these particular values was also provided from single cell recordings in cats that were presented with a stationary bar in the fovea (Ikeda \& Wright, 1975a). Transient cells predominantly responded to spatial frequencies of less than $1 \mathrm{cpd}$ but still showed some response up to $1.5 \mathrm{cpd}$, whereas sustained cells responded across a wide range of frequencies but showed peak responding from 1 to 2 cpd (Ikeda \& Wright, 1975a). Using the 1.0 and $1.75 \mathrm{cpd}$ cutoffs provided some assurance that the segregation of low-and high-frequency-tuned channels would be achieved.

If low spatial frequencies mediate the enhanced processing of patterns with good form, then lowpass filtering should not reduce the global advantage in the manyelement set nor create one in the sparse set. However, highpass filtering at 1.0 and $1.75 \mathrm{cpd}$ should eliminate a global advantage in the many-element set and not produce any effect in the sparse set.

\section{Method}

Subjects. The lowpass condition was performed by 8 subjects, as was the highpass condition. Three subjects in each bandpass condition participated in only one condition. Five subjects participated in both conditions in separate sessions conducted 10 to 15 days apart; 3 subjects performed the highpass condition first, and 2 subjects performed the lowpass condition first. All of the subjects had participated in one or more of the previous experiments. The age range was 24 to 46 years. As in the previous experiments, the subjects reported normal or corrected vision and were paid $\$ 5$ an hour for their participation.

Apparatus. The stimuli were presented on an AppleColor highresolution RGB monitor of a Macintosh IIcx. The screen resolution was 640 dots horizontally $\times 480$ dots vertically, and the monitor utilized an analog input format. The scan rates were $35.0 \mathrm{kHz}$ horizontally and $66.7 \mathrm{~Hz}$ vertically. The mean luminance of the screen was $20 \mathrm{~cd} / \mathrm{m}^{2}$. The Michelson contrast I(max luminance min luminance)/(max luminance + min luminance) $]$ was .94 . The computer also controlled the randomization, presentation, timing, and recording of the subjects' responses. The viewing distance w'as $60 \mathrm{~cm}$. The subjects registered their response by depressing either of two computer keys, S (for square) and D (for diamond). The mapping of the responses to the keys was held constant across tasks and subjects, since square and diamond judgments were analyzed together.

Stimuli. The original square-diamond patterns of the manyelement and sparse sets were subjected to a Fourier analysis that removed spatial frequencies above or below designated values. The convolution was performed in the frequency domain. Frequencies above or below each cutoff were attenuated in separate operations. An inverse transform reconstructed the images. The filtered patterns were transformed to the appropriate format for display on the Mac IIcx system. ${ }^{5}$ Two lowpass versions of each stimulus figure were created by removing frequencies above 1.0 and $1.75 \mathrm{cpd}$. Similarly, two highpass versions were generated by removing frequencies below 1.0 and $1.75 \mathrm{cpd}$. Figure 2 shows a large diamond made of small squares from the many-element and sparse sets after lowpass filtering at 1.0 and $1.75 \mathrm{cpd}$. Figure 3 shows the same configurations after highpass filtering at 1.0 and $1.75 \mathrm{cpd}$

Design. The same identification task was used as in Experiment 2. The lowpass and highpass conditions constituted two subexperiments. Within each sub-experiment, the subjects identified the global or local shape (square or diamond) of many-element and sparse patterns in four separate blocks. Within each block, all four combinations of squares and diamonds were shown equally often. Furthermore, the 1.0- and 1.75 -cpd lowpass patterns were randomly presented in a single block, as were the 1.0- and 1.75-cpd highpass forms. Each run contained 80 trials, permitting 10 repetitions of each combination of shape for two levels of filtering. Thus, in each sub-experiment (lowpass and highpass), there were 320 trials resulting from four trial blocks (global and local judgments for manyelement and sparse patterns).

Procedure. The low- and highpass conditions were conducted in separate sessions, each $30 \mathrm{~min}$ long. The order of presentation of stimulus sets (many-element or sparse) and type of trial (global or local) was counterbalanced within and across subjects

A practice session containing eight trials preceded each block to orient the subjects to the appropriate level and permit them some familiarity with the images. No feedback was provided during prac- 


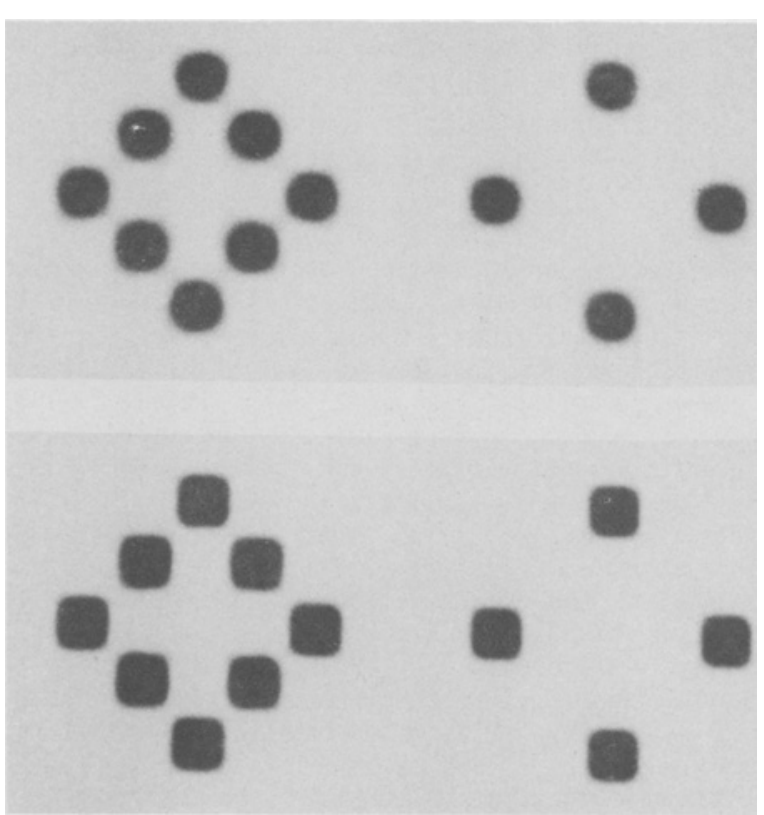

Figure 2. A large diamond made of small squares from the many-element set (left figures) and the sparse set (right figures) after lowpass filtering at $1.0 \mathrm{cpd}$ (top row) and at $1.75 \mathrm{cpd}$ (bottom row) when viewed at approximately $60 \mathrm{~cm}$ (arm's length).

tice. Each trial began with a small fixation point (a small star) for $1 \mathrm{sec}$. The stimulus pattern immediately followed and remained on until the subject responded.

\section{Results and Discussion}

Lowpass figures. Error rates shown in Table 6 (top) averaged $3.2 \%$. An ANOVA of the individual mean error rates [subjects $\times$ stimulus set $\times$ type of trial (global vs. local) $\times$ frequency cutoff] showed a marginally significant interaction for type of trial $\times$ frequency cutoff $[F(1,7)=5.50, p=.0514]$, indicating that the subjects made more errors on patterns filtered at $1.75 \mathrm{cpd}$ when the patterns were sparse than they did on many-element forms. Since RT was also faster for sparse 1.75-cpd patterns, the possibility of a speed-accuracy tradeoff was considered further. If subjects maintain a decision criterion across a block of trials, thereby leading to speed-accuracy tradeoff, the error rates and RTs from each cell should be correlated. The Pearson product-moment correlation on the eight mean values (two stimulus sets $x$ two types of trials $x$ two frequency cutoffs) showed no relationship between error rates and RTs $(r=-.0005)$, ruling out a speed-accuracy tradeoff in these data. Incorrect trials were excluded from further analyses.

The correct mean RTs for global and local responses to lowpass patterns are shown in Table 6 (top). Note that many-element patterns showed a marked global advantage whereas sparse patterns did not. An ANOVA of the individual mean RTs [subjects $\times$ stimulus set $\times$ type of trial (global vs. local) $\times$ frequency cutoff] confirmed that the interaction between stimulus set and type of trial was reliable $[F(1,7)=9.57, p<.02]$. On average, the manyelement set showed a 59-msec global advantage $[F(1,7)=$ $40.83, p<.001]$, whereas the sparse set showed a nonsignificant 6-msec global-local difference $(F<1)$. The significant levels were sufficient for multiple follow-up tests (modified Bonferroni test). These data show that many-element patterns subjected to lowpass filtering retained the global advantage found in unfiltered forms. Moreover, the global-local difference in lowpass sparse patterns was not reliable, also replicating the pattern of results obtained with unfiltered forms.

Some evidence for dependency between spatial frequencies and global-local processing within the 1.0 and $1.75 \mathrm{cpd}$ range is revealed by the interaction between type of trial and frequency cutoff $[F(1,7)=8.56, p<.03]$. Local processing times were slower as more high frequencies were removed [1.0-cpd frequency cutoff; $F(1,7)=$ $21.53, p<.01$ ], whereas global RTs showed little change $(F<1)$, suggesting that small bandpass changes of intermediate spatial frequencies were sufficient to affect local processing but were unimportant to global processing. Adjustment by a modified Bonferroni procedure showed that the reported significant levels were adequate. The only other reliable effect was the main effect for type of trial $[F(1,7)=33.68, p<.001]$, which showed that, on average, local RT was slower than global RT. This effect is probably subsumed by the previous interactions.

In summary, one prediction from the spatial frequency hypothesis has been confirmed: Lowpass filtering of square-diamond patterns did not disrupt the global advantage found in the previous experiments. That is, many-

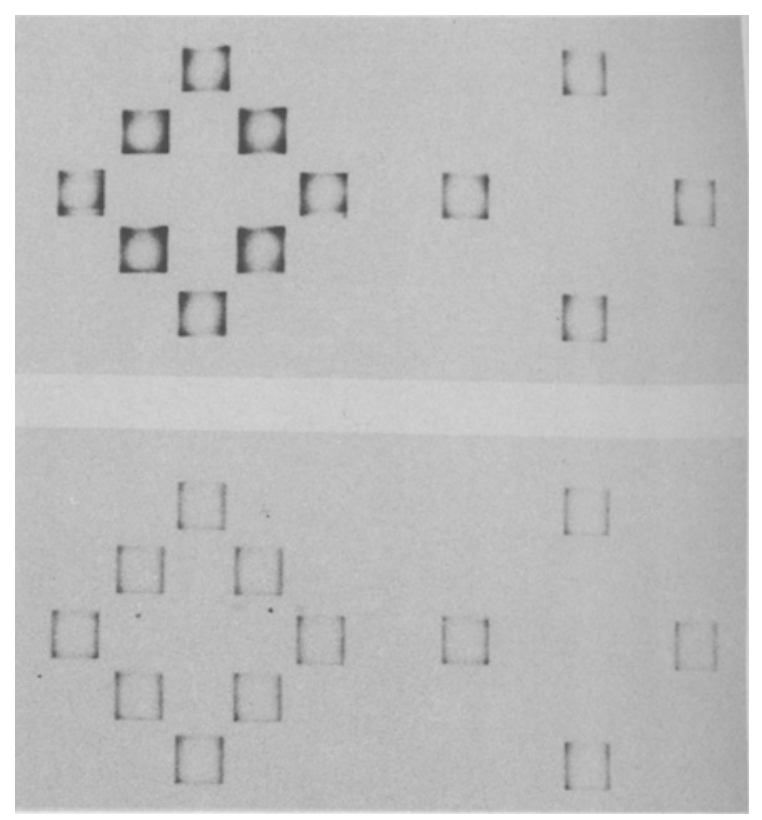

Figure 3. A large diamond made of small squares from the manyelement set (left figures) and the sparse set (right figures) after highpass filtering at $1.0 \mathrm{cpd}$ (top row) and at $1.75 \mathrm{cpd}$ (bottom row) when viewed at approximately $60 \mathrm{~cm}$ (arm's length). 
Table 6

Mean Global and Local Response Times (RTs, in Milliseconds) for Patterns Lowpass and Highpass Filtered at 1.00 and 1.75 cpd by Stimulus Set

\begin{tabular}{|c|c|c|c|c|c|c|c|}
\hline \multirow{3}{*}{$\begin{array}{l}\text { Stimulus } \\
\text { Set }\end{array}$} & \multirow{3}{*}{$\begin{array}{l}\text { Cutoff } \\
\text { (cpd) }\end{array}$} & \multicolumn{4}{|c|}{ Type of Trial } & \multirow{2}{*}{\multicolumn{2}{|c|}{$\begin{array}{c}\text { Global } \\
\text { Advantage* }\end{array}$}} \\
\hline & & \multicolumn{2}{|c|}{ Local } & \multicolumn{2}{|c|}{ Global } & & \\
\hline & & $M$ & ER & $M$ & ER & $M$ & $S E$ \\
\hline \multicolumn{8}{|c|}{ Lowpass Filtered Patterns } \\
\hline \multirow[t]{3}{*}{ Many } & 1.00 & 466 & $3.8 \%$ & 401 & $3.2 \%$ & $65+$ & 23 \\
\hline & 1.75 & 459 & $1.9 \%$ & 406 & $1.9 \%$ & $53 \dagger$ & 25 \\
\hline & Mean & 462 & $2.8 \%$ & 403 & $2.6 \%$ & $59+$ & 9 \\
\hline \multirow[t]{3}{*}{ Sparse } & 1.00 & 434 & $3.2 \%$ & 416 & $2.9 \%$ & 18 & 34 \\
\hline & 1.75 & 414 & $2.9 \%$ & 420 & $5.7 \%$ & -6 & 23 \\
\hline & Mean & 424 & $3.0 \%$ & 418 & $4.3 \%$ & 6 & 11 \\
\hline \multicolumn{8}{|c|}{ Highpass Filtered Patterns } \\
\hline \multirow[t]{3}{*}{ Many } & 1.00 & 530 & $4.4 \%$ & 508 & $2.2 \%$ & 22 & 80 \\
\hline & 1.75 & 517 & $4.7 \%$ & 523 & $2.5 \%$ & -5 & 66 \\
\hline & Mean & 524 & $4.6 \%$ & 516 & $2.4 \%$ & 8 & 31 \\
\hline \multirow[t]{3}{*}{ Sparse } & 1.00 & 549 & $2.5 \%$ & 536 & $3.5 \%$ & 13 & 70 \\
\hline & 1.75 & 536 & $3.2 \%$ & 525 & $5.0 \%$ & 11 & 55 \\
\hline & Mean & 542 & $2.8 \%$ & 530 & $4.2 \%$ & 12 & 26 \\
\hline
\end{tabular}

Note-ER $=$ error rates. $\quad{ }^{*}$ Local RT minus global RT. $\quad t p<.02$.

element patterns showed a reliable global advantage, but sparse patterns did not. In addition, there was evidence that local RT was more disrupted by removal of high spatial frequencies than was global RT.

Highpass forms. Error rates presented in Table 6 (bottom) averaged $3.5 \%$. An ANOVA of the individual mean error rates [subjects $\times$ stimulus set $\times$ type of trial (global vs. local) $\times$ frequency cutoff] showed no reliable main effects $[F(1,7)<4.76, p>.07$, in all cases] or interactions $[F(1,7)<1.91, p>.21$, in all cases], indicating that a speed-accuracy tradeoff was not occurring. Incorrect trials were excluded.

The correct global and local RTs for highpass figures are shown in Table 6 (bottom) ${ }^{6}$. Unlike in the lowpass patterns, there was no evidence for a global advantage in the many-element patterns. An ANOVA of the individual mean RTs [subjects $\times$ stimulus set $\times$ type of trial (global vs. local) $\times$ frequency cutoff] confirmed that the interaction between stimulus set and type of trial was not reliable $(F<1)$. In the many-element set, global RT was $8 \mathrm{msec}$ faster than local RT $(F<1)$. Similarly, sparse patterns showed a 12-msec global-local difference $(F<1)$. None of the main effects $(F<1$ in all cases $)$ and the other interactions $[F(1,7)<4.85, p>.06$, in all cases] were significant.

In summary, highpass patterns showed no reliable global advantage, supporting the prediction that low spatial frequencies are necessary for a global precedence effect to occur in many-element forms. As expected, removing low spatial frequencies did not change the outcome of the sparse set: Neither unfiltered or highpass sparse patterns showed a global or a local advantage.

The hypothesis that low spatial mechanisms mediate the association between pattern goodness and global precedence is supported by the findings that a global advantage occurred only in unfiltered patterns and in those with intact low spatial frequencies. When the low-frequency band (less than $1 \mathrm{cpd}$ ) was removed, the global advantage was lost. ${ }^{7}$ Note that Navon had also appealed to a spatial frequency explanation for global precedence. In fact, he suggested that low spatial frequencies could fully account for global precedence (Navon \& Norman, 1983) because the global form was always larger than the local elements. The results from sparse patterns argue against Navon's hypothesis. Although unfiltered and lowpassfiltered sparse patterns contained intact low-frequency bands, a global advantage did not occur, suggesting that low spatial frequencies are a necessary but not a sufficient determinant of global precedence.

The present results indicate that the goodness of the global form is conducted by low spatial frequencies. An examination of two-dimensional (2-D) amplitude spectra of many-element and sparse patterns (from the Fourier analysis) suggests a possible explanation for how pattern goodness can be represented. The main characteristic distinguishing squares and diamonds is the orientation of the lines bounding the figure. Several studies have found that early visual mechanisms are tuned not only to a narrow band of spatial frequencies but also to a specific orientation (e.g., Breitmeyer \& Gantz, 1976; Graham, 1992; Ikeda \& Wright, 1975b). The claim that both dimensions are necessary for the occurrence of a global precedence effect is consistent with findings that maximal activation of these mechanisms requires a unique combination of both attributes (Greenlee \& Magnussen, 1988; Hughes, 1986). ${ }^{8}$ In the 2-D spectra of many-element patterns, the distribution of power within the low spatial frequency band (greater than $.5 \mathrm{cpd}$ but less than $1 \mathrm{cpd}$ ) was concentrated in the same orientation as the global pattern and thus was distinctive. For sparse patterns, however, the power was diffused and more ambiguous with respect to orientation. 
To illustrate this distinction, the amplitude values of a horizontal and diagonal cross-section were extracted from the Fourier analysis of the many-element and sparse patterns used in Experiment 1A. The values were multiplied by their frequency to amplify the low power of the higher frequencies and were combined into four groups on the basis of the stimulus set and the global form (within each stimulus set, patterns with the same global form but different local forms were combined): many-element, global squares; many-element, global diamonds; sparse, global squares; and sparse, global diamonds. In Figure 4 , the combined amplitude values are shown for the horizontal and diagonal dimension for each group. The plots are restricted to low spatial frequencies (less than $1 \mathrm{cpd}$ ), since the observed orientation differences only occurred in this range. Note that in many-element patterns, relative amplitude above $0.5 \mathrm{cpd}$ is greater in the orientation congruent with the global form. But in sparse forms, the distribution of amplitude is more ambiguous with respect to the orientation of the global form. However, the values from 0.1 to $.4 \mathrm{cpd}$, particularly in the sparse plots, do not fully support this observation. But inconsistencies in this range may not be perceptually salient because the lower limit of the contrast sensitivity function in the human visual system is $0.5 \mathrm{cpd}$.

The present findings are consistent with this analysis. If there is no low-frequency input (i.e., highpass patterns), or the low-frequency input contains insufficient orientation information (i.e., sparse figures), then visual mechanisms responsible for transmitting blobs or directing attention may be inactive and a global advantage cannot occur. The global and local shapes are recognizable in both instances, but the processing advantage for global information does not occur.

A reasonable prediction from this conclusion is that the perceived pattern goodness of many-element patterns should vary with its spatial frequency content. The next experiment examined whether the subjects would judge

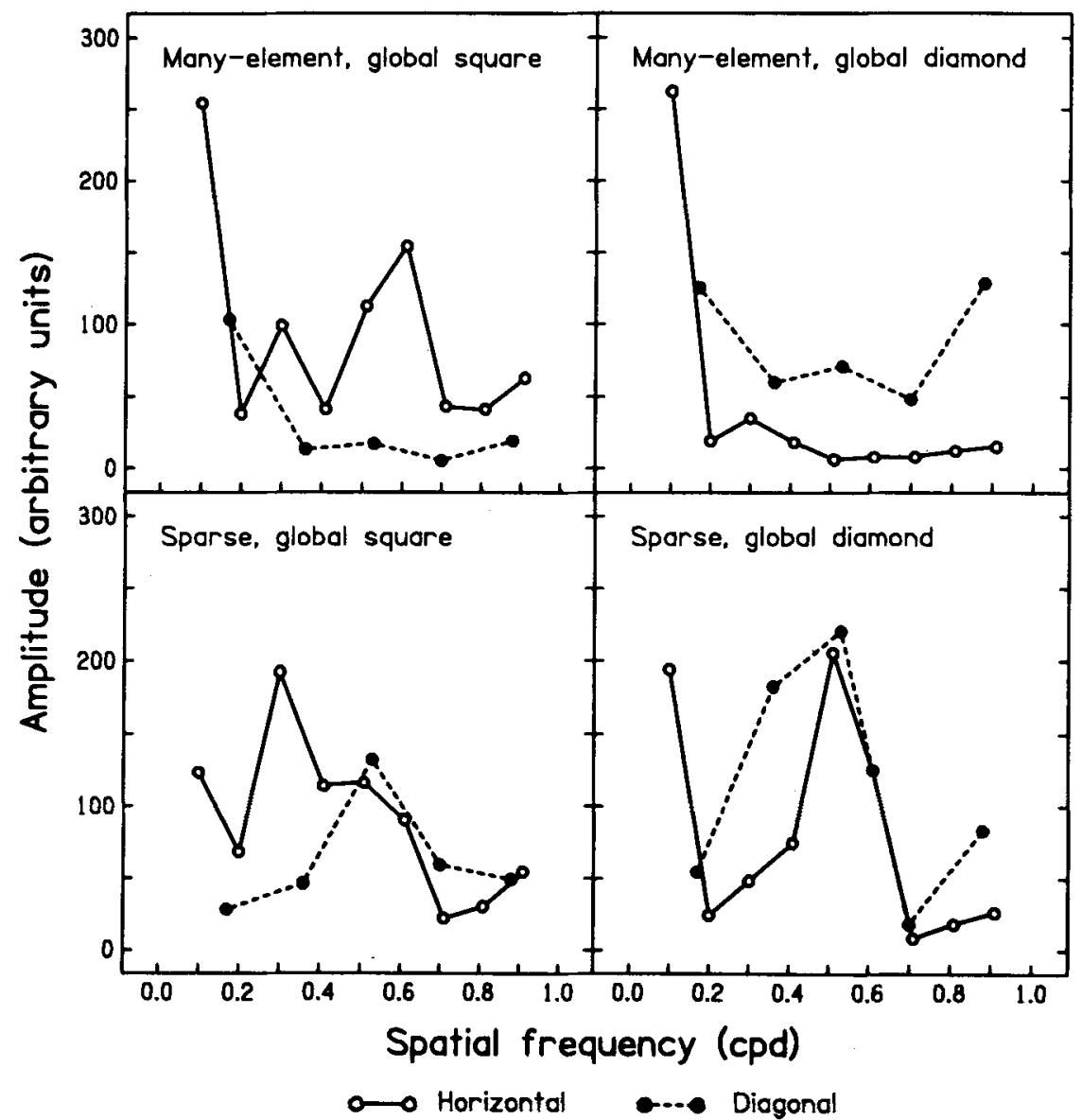

Figure 4. Two-dimensional amplitude plots of many- and few-element patterns grouped by global form. The plotted values are a horizontal and right diagonal cross-section of spatial frequencies lower than 1 cpd extracted from the Fourier analyses of the unfiltered square-diamond figures. 
many-element configurations as more recognizable when they contained only low frequencies than when they contained only high frequencies.

\section{EXPERIMENT 3B}

Experiment 3A has shown that a global advantage requires both a "good" global form and the presence of low spatial frequencies. It was posited that pattern goodness (orientation input) might be conveyed by lowfrequency mechanisms. It follows, then, that manyelement patterns should be judged as less "good" if low spatial frequencies were removed. Removing high spatial frequencies, however, should not affect the perceived goodness of the global form. Similar but opposite predictions can be made for the local form. Removing high spatial frequencies should reduce the perceived pattern goodness of local forms, whereas removing low spatial frequencies should have little effect.

\section{Method}

Subjects. The subjects were 8 paid adults who reported normal or correct-to-normal vision. All had participated in one or more of the previous experiments and 5 had performed the goodnessrating task of Experiment 1A. The age range was 24 to 44 years.

Stimuli. Three types of many-element patterns were used in this experiment: (1) unfiltered forms, shown in Figure 1, (2) lowpass forms filtered at $1.75 \mathrm{cpd}$, shown in Figure 2, and (3) highpass forms filtered at $1.75 \mathrm{cpd}$, shown in Figure 3.

Design. Global and local goodness ratings were obtained by the same method used in Experiments 1A. On each trial, the subjects were informed of the upcoming shape by the words "square" or "diamond" centered near the top of the screen for $1 \mathrm{sec}$. A pair of stimulus patterns that differed only in spatial frequency composition were then presented. The subjects chose which pattern had the better shape. All combinations of the three stimulus types were presented equally often, with the left-right position of the pair counterbalanced across trials. The goodness rating task had 80 trials and was presented twice to obtain global and local judgments.

Procedure. The apparatus and set up were identical to Experiment 3A. The subjects were shown examples of the stimulus patterns and given a practice run of 12 trials before the experimental data were collected. The level of focus was counterbalanced across subjects. The subjects pressed the " $X$ " key to choose the display on the left or the "." key to choose the display on the right.

\section{Results and Discussion}

The global form containing low spatial frequencies was judged as easier to encode. Unfiltered patterns were judged as easier to encode than highpass forms $82 \%$ of the time. Moreover, lowpass patterns were judged as easier to encode than highpass forms $80 \%$ of the time. These values exceeded chance in both instances $[t(7)=6.43$, $p<.001$, and $t(7)=3.36, p<.02$, respectively]. By contrast, when comparing unfiltered and lowpass patterns, the subjects were equally likely to choose the lowpass forms $(55 \%$ of the trials) or the unfiltered forms $(t<1)$. In sum, both unfiltered and lowpass patterns were rated higher in patterns goodness than were highpass forms, supporting the hypothesis that the recognizability of global information is determined by low-frequency form information.
The local form containing high spatial frequencies was judged as easier to encode. Unfiltered patterns were chosen as easier to recognize $100 \%$ of the time when compared with lowpass forms. By contrast, the subjects chose the unfiltered patterns on $68 \%$ of the trials when the patterns were compared with highpass forms, but this difference did not exceed chance $[t(7)=1.97, p=.09]$. When the subjects compared low and highpass forms, they chose highpass patterns $94 \%$ of the time $[t(7)=5.33$, $p<.01]$. The equivalence in pattern goodness between the local forms of unfiltered and highpass patterns suggests that the recognizability of the local elements involved spatial frequencies above $1.75 \mathrm{cpd}$.

The findings of Experiment $3 \mathrm{~A}$ can be explained by the present results. A global advantage occurred only in global patterns perceived as having good form. Furthermore, the perception of global good form appeared to be conveyed by the low spatial frequency band of the stimulus.

\section{GENERAL DISCUSSION}

This series of experiments demonstrated the dependence of global precedence on the goodness of the pattern, which in turn relies on low spatial frequencies. Contrary to the Navon study (1983), a global advantage is eliminated when the number of local elements is sparse. The small size of Navon's stimuli could account for the preservation of a global advantage in his few-element patterns. Patterns as small as those used by Navon yielded a reliable global advantage whether they were made of eight or four local elements. Furthermore, the subjects' ratings indicated that the local shapes of small patterns were hard to discriminate, thereby enhancing the global advantage even when the global form was poor.

The global advantage shown by the "good," manyelement patterns was eliminated when low spatial frequencies were removed, suggesting that low-frequency-tuned visual mechanisms convey information about pattern goodness. It is possible that the perception of good form in these patterns may be associated with the distinctiveness of low-frequency orientation information. The removal of high frequencies did not interfere with the generation of a global advantage. As predicted, the subjects' ratings of the pattern goodness of many-element configurations reflected the spatial frequency manipulation: Forms with low spatial frequencies were rated higher than those with only high spatial frequencies. Although the presence of low-frequency information is necessary, it is not sufficient for a global precedence effect. The cooccurrence of good form and low spatial frequencies was required to elicit a global advantage.

Much research on global precedence has centered on two controversies: (1) the robustness of the effect and (2) the locus (stage-of-processing) for the effect. This study provides new evidence that global precedence effects are robust. The effects of sparsity on the global advantage were replicated in an identification task and in a same-different task, whether attention was unrestricted or focused on one level with an instruction to ignore the 
other. On the other hand, the present data do not resolve the stage-of-processing question. The symmetric interference effects of Experiment 2 rule out a strict early encoding model and suggest a combination of faster encoding and postperceptual processes. The roughly uniform interference effects in all stimulus sets regardless of the co-occurrence of a global advantage suggest that interference effects might be independent of the global advantage, in which case the locus issue becomes wholly indeterminate in these data.

There is an alternative perceptual explanation for the observed association between good form and global precedence. It is possible that many-element patterns generate a strong axis that entrains the local elements (see Marr, 1982, p. 327). The vertical/horizontal axes of the square configurations would not alter the perceived orientation of local elements. But in diamond configurations, the diagonal axes can render the local elements ambiguous and slow local processing time to create an apparent global precedence effect. The highpass filtered, many-element patterns might not show this effect if low spatial frequencies mediate the entrainment (see Carrasco et al., 1986, for an example of the role of low frequencies in other Gestalt phenomena). To test for this possibility, the data in Experiments 1A, 2, and 3A were recoded by the global configuration and reanalyzed to determine if only manyelement, diamond configurations show a global advantage. The global advantage for square and diamond configurations in each stimulus set and experiment is shown in Table 7. Separate analyses for each experiment showed that square as well as diamond configurations yielded a reliable global advantage for many-element forms (except when highpass filtered). ${ }^{9}$ Local form ambiguity might explain why the global advantage in the many-element patterns of Experiment 1 was about $65 \mathrm{msec}$ larger in diamond as opposed to square configurations. But this effect was not replicated in Experiments 2 and 3A, and a similarly larger global advantage also occurred in sparse patterns even though the sparse configurations were not predicted to entrain local forms. The important result of these analyses is that local form ambiguity cannot wholly account for the effects of sparsity on global precedence.

Table 7

Mean Global Advantage* (in Milliseconds) for Square and Diamond Configurations by Stimulus Set and Experiment

\begin{tabular}{|c|c|c|c|c|}
\hline \multirow[b]{2}{*}{ Experiment } & \multirow[b]{2}{*}{ Filter } & \multirow[b]{2}{*}{ Stimulus Set } & \multicolumn{2}{|c|}{ Configuration } \\
\hline & & & Square & Diamond \\
\hline \multirow[t]{3}{*}{1} & & Many & 81 & 148 \\
\hline & & Sparse & -7 & 65 \\
\hline & & Dense & 22 & -15 \\
\hline \multirow[t]{3}{*}{2} & & Many & 45 & 57 \\
\hline & & Sparse & -6 & -28 \\
\hline & & Dense & -5 & -45 \\
\hline \multirow[t]{4}{*}{3} & Lowpass & Many & 60 & 59 \\
\hline & & Sparse & 21 & -10 \\
\hline & Highpass & Many & 9 & 6 \\
\hline & & Sparse & 19 & 5 \\
\hline
\end{tabular}

*Global advantage equals local RT minus global RT.
In summary, a processing advantage for global information seems to depend on the goodness of the global configuration, and this information is conveyed by low spatial frequencies. The conjunction of both stimulus attributes, goodness and low spatial frequency information, may be required to activate fast-conducting visual mechanisms tuned to low spatial frequencies. However, the faster encoding of low-frequency information may be secondary to its capacity to attract processing resources.

\section{REFERENCES}

Bassi, C. J., \& LehmKuhle, S. (1990). Clinical implications of parallel visual pathways. Journal of the American Optometric Association, 61, 98-110

BeLl, H. H., \& HANDEL, S. (1976). The role of pattern goodness in the reproduction of backward masked patterns. Joumal of Experimental Psychology: Human Perception \& Performance, 2, 139-150.

BoER, L. C., Keuss, P. J. G. (1982). Global precedence as a postperceptual effect: An analysis of speed-accuracy tradeoff functions. Perception \& Psychophysics, 31, 358-366

BREITMEYeR, B. G., GANZ, L. (1976). Implications of sustained and transient channels for theories of visual pattern masking, saccadic suppression, and information processing. Psychological Review, 83 , 1-36.

Carrasco, M., Figueroa, J. G., \& Willen, D. (1986). A test of the spatial-frequency explanation of the Müller-Lyer illusion. Perception, $15,553-562$.

Checkosky, S. F., \& Whitlock, D. (1973). Effects of pattern goodness on recognition time in a memory search task. Journal of $E x-$ perimental Psychology, 100, 341-348.

GARNER, W. R., \& SutlifF, D. (1974). The effect of goodness on encoding time in visual pattern discrimination. Perception \& Psychophysics, 16, 426-430.

Graham, N. (1992). Breaking the visual stimulus into parts. Current Directions in Psychological Science, 1, 55-61.

GreENLEE, M. W., \& MAGNUSSEN, S. (1988). Interactions among spatial frequency and orientation channels adapted concurrently. Vision Research, 28, 1303-1310.

Grice, G. R., Canham, L., \& Boroughs, J. M. (1983). Forest before trees? It depends where you look. Perception \& Psychophysics, 33 , 121-128.

HoffmaN, J. E. (1980). Interaction between global and local levels of a form. Journal of Experimental Psychology: Human Perception \& Performance, 6, 222-234

Hughes, H. C. (1986). Asymmetric interference between components of suprathreshold compound gratings. Perception \& Psychophysics, 40, 241-250.

Hughes, H. C., Fendrich, R., \& Reuter-Lorenz, P. A. (1990). Global versus local processing in the absence of low spatial frequencies. Journal of Cognitive Neuroscience, 2, 272-282.

Hughes, H. C., LAyton, W. M., Baird, J. C., \& Lester, L. S. (1984). Global precedence in visual pattern recognition. Perception \& Psychophysics, 35, 361-371.

IKEDA, H., \& WRIGHT, M. J. (1975a). Spatial and temporal properties of "sustained" and "transient" neurones in area 17 of the cat's visual cortex. Experimental Brain Research, 22, 363-383.

IKEDA, H., \& WRIGHT, M. J. (1975b). Retinotopic distribution, visual latency and orientation tuning of "sustained" and "transient" cortical neurones in area 17 of the cat. Experimental Brain Research, 22, 385-398.

KINCHLA, R. A., \& WolfE, J. M. (1979). The order of visual processing: "Top-down," "bottom-up," or "middle-out." Perception \& Psychophysics, 25, 225-231.

KoEhler, W. (1947). Gestalt psychology. New York: Liveright.

LAMB, M. R., \& RoberTson, L. C. (1989). Do response time advantage and interference reflect the order of processing of global- and local-level information? Perception \& Psychophysics, 46, 254-258.

Livingstone, M. S., \& HubeL, D. H. (1987). Psychophysical evidence 
for separate channels for the perception of form, color, movement, and depth. Journal of Neuroscience, 7, 3418-3468.

MARR, D. (1982). Vision. New York: W. H. Freeman.

MARTIN, M. (1979). Local and global processing: The role of sparsity. Memory \& Cognition, 7, 476-484.

MilLER, J. (1981). Global precedence in attention and decision. Journal of Experimental Psychology: Human Perception \& Performance, 7, 1161-1174.

MülLER, H. J., \& RABBitT, P. M. A. (1989). Reflexive and voluntary orienting of visual attention: Time course of activation and resistance to interruption. Journal of Experimental Psychology: Human Perception \& Performance, 15, 315-330.

Navon, D. (1977). Forest before trees: The precedence of global features in visual perception. Cognitive Psychology, 9, 353-383.

Navon, D. (1981). The forest revisited: More on global precedence. Psychological Research, 43, 1-32.

NAvoN, D. (1983). How many trees does it take to make a forest? Perception, 12, 239-254.

Navon, D. (1991). Testing a queue hypothesis for the processing of global and local information. Journal of Experimental Psychology: General, 120, 173-189.

Navon, D., \& Norman, J. (1983). Does global precedence really depend on visual angle? Joumal of Experimental Psychology: Human Perception \& Performance, 9, 955-965.

Paquet, L., \& MerikLe, P. M. (1984). Global precedence: The effect of exposure duration. Canadian Journal of Psychology, 38, 45-53.

Petersik, J. T. (1978). Possible role of transient and sustained visual mechanisms in the determination of similarity judgments. Perceptual \& Motor Skills, 47, 683-698.

Pomerantz, J. R. (1983). Global and local precedence: Selective attention in form and motion perception. Journal of Experimental Psychology: General, 112, 516-540.

RoberTson, L. C. (1986). From Gestalt to neo-Gestalt. In T. J. Knapp \& L. C. Robertson (Eds.), Approaches to cognition: Contrasts and controversies (pp. 165-166). Hillsdale, NJ: Erlbaum.

Robertson, L. C., \& LAMB, M. R., \& KNIGHT, R. T. (1988). Effects of lesions of temporal-parietal junction on perceptual and attentional processing in humans. Journal of Neuroscience, 8, 3757-3769.

Shulman, G. L., Sullivan, M. A., Gish, K., \& Sakoda, W. J. (1986). The role of spatial-frequency channels in the perception of local and global structure. Perception, 15, 259-273.

ShUlman, G. L., \& WILSON, J. (1987). Spatial frequency and selective attention to local and global information. Perception, 16. 89-101.

TODD, J. T., \& VAN GELDER, P. (1979). Implications of a transientsustained dichotomy for the measurement of human performance. Journal of Experimental Psychology: Human Perception \& Performance, 5, 625-638.

WARD, L. M. (1982). Determinants of attention to local and global features of visual forms. Journal of Experimental Psychology: Human Perception \& Performance, 8, 562-581.

Ward, L. M. (1983). On processing dominance: Comment on Pomerantz. Joumal of Experimental Psychology: General, 112, 541-546.

\section{NOTES}

1. In Experiment 1 of the Navon and Norman (1983) study, subjects showed a slightly larger global advantage when they performed a divided versus a selective-attention task.
2. The observed redundancy gain could be interpreted in two ways: (1) global and local information are encoded in parallel and contribute to a joint decision process (interlevel facilitation), and (2) local information is occasionally encoded faster than global information. These alternatives were tested by calculating the cumulative density functions for each stimulus set (see Miller, 1981, for a full description of these procedures). In this analysis, the cumulative probability of the fastest values for trials with global and local differences was compared with the fastest values for trials having either global or local differences. In all cases, there was no reliable advantage for differences at both levels compared with differences on one level alone. In short, these data do not show interlevel facilitation but support the claim that local processing is sometimes faster than global processing.

3. Although experienced subjects were aware that the overall objec tive of the study was to examine factors that influence pattern recognition, they were not told the specific predictions concerning the manipulation of sparsity, attention, or type of response required.

4 . The trend shown by few-element patterns-small, unreliable local precedence effects and a tendency toward more local interference with global than vice versa-invites speculation that encoding differences (as determined by consistency effects) might account for local processing time.

5. The images were not corrected for the nonlinearity of the monitor.

6. The longer RT found in the highpass condition might suggest that highpass patterns were inherently more difficult to process than were lowpass patterns. However, the higher mean RT can be attributed to the 3 subjects who only performed the highpass condition. The pattern of results was the same for the slow subjects as it was for the 5 others. Moreover, replication studies with a new sample showed similar mean $\mathrm{RT}$ in the low- and highpass patterns.

7. The lowpass and highpass data were reanalyzed together to test whether the subjects who performed in both conditions differed from those who performed in only one condition. Although all subjects had participated in one or more previous experiments, it is possible that carryover effects from experience with the altemate bandpass condition confounded the results. An ANOVA was performed on the individual mean global advantage in many-element and sparse forms $\times$ the betweensubject factors, bandpass condition and experience. The results yielded no significant main and interaction effects for experience $[F(1,12)<$ $2.77, p>.13]$. Both experienced and inexperienced subjects showed a global advantage only in lowpass, many-element patterns.

8. These data do not rule out other stimulus factors that could affect global precedence. Ward (1983) suggested that any factor that changes the relative discriminability between global and local information will influence which level shows processing dominance. Apart from manipulations of size (spatial frequency) or good form (orientation), global-local processing is sensitive to changes in brightness (Boer \& Keuss, 1982; Hughes, Layton, Baird, \& Lester, 1984) and variation in the similarity relations between the target and distractor shapes (Lamb \& Robertson, 1989).

9. The divided-and selective-attention data were combined and error rate data were excluded, since these added little new information. The details of these analyses are omitted here but may be obtained from the author.

(Manuscript received October 15, 1991; revision accepted for publication June 21,1992 .) 